\title{
Regions Segmentation from SAR Images
}

\author{
Luigi Cinque $^{1}$ and Rossella Cossu ${ }^{2}$ \\ 1 Universitá degli Studi Sapienza, Roma \\ 2 Istituto per le Applicazioni del Calcolo-CNR, Roma \\ r.cossu@iac.cnr.it
}

\begin{abstract}
In this paper, we propose an approach based on the level set method for segmenting SAR (Synthetic Aperture Radar) images. In particular, the segmentation process presented consists in the evolution of an initial curve, including the interested region, until it reaches the boundary of the area to be extracted. The procedure proposed allows to obtain the same result of segmentation independently of the initial position of the curve. The results are shown on both synthetic and real images. The analyzed images are SAR PRI (Precise Images), acquired during the mission ERS2.
\end{abstract}

Keywords: Level set, Image segmentation, SAR, Speckle noise.

\section{Introduction}

Image segmentation plays a fundamental role in the SAR image interpretation. The segmentation of SAR images is usually recognized as a complex problem, because of multiplicative noise, called speckle, which produces grainy images. Recently, a number of methods based on curve evolution have been proposed for segmentation of SAR images. In this paper we present a procedure based on the evolution of curves, described by the level set method, to extract distinct regions from SAR images. This method, proposed by Osher and Sethian [12]3], is based on the identification of an area of interest as the zero level set of an implicit function that evolves according to a PDE (partial differential equation) model with an appropriate speed function.

SAR images present advantages and disadvantages; the acquired images provide information under varied weather conditions, during night as well as day. On the other hand SAR images present a granular effect due to the presence of the speckle noise.

The segmentation methods based on edge detection filters, often show edges which may not form a set of closed curves surrounding connected regions. The traditional techniques of histogram thresholding and region-based need a preprocessing based on speckle reduction. Moreover, the region-growing techniques have the limit of depending on the selection of the starting points.

The segmentation process proposed starts from an initial curve (zero level set) defined on the image that evolves until it stops at the contour of the interest object. The evolution of the initial curve is determined by a speed function, which is a fundamental choice to achieve a good segmentation. 
In this paper two different speed functions are introduced and their results compared with a series of tests on synthetic SAR images. In particular a first approach developed is based on the assumption that each region to segment through level set is modeled by a Gamma distribution. In this case an expression of propagation speed of the front is obtained by computing intensity averages of the regions; the method does not need to reduce speckle noise [5].

In the second approach developed the speed function is based on the computation of image gradient and takes into account the problem of filtering speckle noise in the image, which was faced with the application of the SRAD (Speckle Reducing Anisotropic Diffusion) technique to SAR image [6] 7] 8].

Finally we propose a combined speed, based on the contributes of the previous speeds functions. In this case the proposed procedure has the important peculiarity to extract the same contour from an image also starting from different initial contours. Our approach is validated and compared using a series of tests on synthetic SAR images. These tests demonstrate our method allows to obtain the same result of segmentation independently of the initial position of the curve.

The SAR PRI image here segmented has been acquired during ERS2 mission. ERS2 SAR system is capable of $25 \mathrm{~m}$. resolution from an altitude of $800 \mathrm{~km}$, at radar wavelength of $5.7 \mathrm{~cm}$.

The paper is organized as follows. In Section 2, the level set method is briefly described. In Section 3, speed computation related to level set method and noise reducing are presented. In Section 4 experimental results and applications to SAR image are shown. Some conclusions are drawn in Section 5.

\section{Level Set Approach}

It should be mentioned that the segmentation through the level set method has become very popular over the last decades. In particular, this methodology describes the evolution of an initial curve, including the interested region, until it reaches the boundary of the area to be extracted.

Let $I: \Omega \rightarrow \Re^{n}$ be the intensity image function where $\Omega \subset \Re^{2}$.

The goal of image segmentation is to partition $\Omega$, moving from image $I$, in order to extract disjoint regions covering $\Omega$.

The boundary of the region of interest may be considered as a curve belonging to a family in which the time evolution is described by the following level set equation

$$
\frac{\partial \Phi(\mathbf{x}(t))}{\partial t}+F(\mathbf{x}(t))|\nabla \Phi(\mathbf{x}(t))|=0
$$

where $F(\mathbf{x}, t)$, representing the curve speed in the normal direction, is related to the image features. The main advantages of using the level set is that complex shaped regions can be detected and handled implicitly. The initial curve (zero level set) evolves until it stops at the contour of the interest object. 
For the numerical approximation of the level set equation in a domain $\Omega \subset \Re^{2}$ we introduce the computational domain $\Omega^{*}$ obtained by considering a uniform partition of $\Omega$ in $(N-1) \times(M-1)$ disjoint rectangles $\Omega_{i j}$ with edges $\Delta x=\Delta y$, usually in an image $\Delta x=\Delta y=1$. Let $P_{i, j} \equiv P\left(x_{i}, y_{j}\right) \quad i=1, \ldots, N ; j=1, \ldots, M$ a point in $\Omega^{*}$ and $\phi_{i, j}^{n}$ the value of the function $\phi(\mathbf{x}(t))$ at $P_{i, j}$ at time $t^{n}$. Let $v(\mathbf{x}(t))$ be the speed function: the algorithm starts by initializing $\phi(\mathbf{x}(t))$ as a signed distance function

$$
\phi(\mathbf{x}(0))= \pm d
$$

where

$$
d(\bar{x})=\min _{\bar{x}_{\gamma} \in \gamma}\left|\bar{x}-\overline{x_{\gamma}}\right| .
$$

Now, known the value of $\phi_{i, j}^{n}$, the value $\phi_{i, j}^{n+1}$ is computed by a 2 -order ENO scheme with the TVD (Total Variation Diminishing) Runge Kutta scheme for the time integration.

We underline that the definition of $\phi(\mathbf{x}(t))$ as a signed distance function is crucial. In fact, during the evolution the level set function does not remain a signed distance function; so that it is necessary to re-initialize the algorithm at regular intervals in order to limit numerical dissipation. Moreover the choice of speed function is a fundamental task for this segmentation approach.

\section{Combined Speed}

As mentioned above, the level set method starts from the definition of an initial curve in the domain of the image. In our case, the initial curve on the SAR images is placed in the background zone, so that it surrounds the object of interest. The evolution of the initial curve is determined by a speed function, of fundamental importance to achieve a good segmentation.

In this section we present an combined speed obtained by the contributes of the two speed functions, here introduced and compared. The first, called averagebased speed, is a function based on modeling the intensity of image by a Gamma distribution. The second, called gradient-based speed, is a function based on the computation of image gradient.

In this work the goal of the segmentation process is to extract two types of regions $\left.R_{i} \quad i \in\{1,2\}\right)$ representing objects and background.

Let be $I(\mathbf{x}(t))$ the SAR image intensity which we model by a Gamma distribution. After some probabilistic considerations and algebraic manipulations we obtain the average-based speed given

$$
v(\mathbf{x}(t))=\frac{d \gamma}{d t}=-\left(\log \mu_{R_{1}}+\frac{I(\mathbf{x}(t))}{\mu_{R_{1}}}-\log \mu_{R_{2}}-\frac{I(\mathbf{x}(t))}{\mu_{R_{2}}}+\lambda k\right)
$$


where a $\lambda k$ is a regularization term, with $\lambda$ a positive real constant and $k$ the mean curvature function, $\mu_{R_{i}}$ is the mean intensity given

$$
\mu_{R_{i}}=\frac{\int_{R_{i}} I(\mathbf{x}(t)) d \mathbf{x}}{a_{R_{i}}}
$$

and where the area $a_{R_{i}}$ is given

$$
a_{R_{i}}=\int_{R_{i}} d \mathbf{x}
$$

The implementation of the level set method with the speed based on regions means detects the object with more precision in terms of pixels than the next one since the image is not dealt with filters for noise reduction. We observed that the best result is obtained by locating the initial curve as nearly as possible to the region of interest, so that the final result depends on the position of the starting curve.

It is well known that in images corrupted by strong noise, the computation of gradient could detect false edges. Because the SAR images are affected by speckle noise, they are pre-processed by means of the SRAD algorithm which is an extension of Perona-Malik algorithm 6 6 7

$$
\left\{\begin{array}{l}
\frac{\partial I(\mathbf{x}(t))}{\partial t}=\nabla \cdot[c(q) \nabla I(\mathbf{x}(t))] \\
I(\mathbf{x}(0))=I_{0}
\end{array}\right.
$$

where the diffusion coefficient is

$$
c(q)=\frac{1}{1+\left[q^{2}(\mathbf{x}(t))-q_{0}^{2}(t)\right] /\left[q_{0}^{2}(t)\left(1+q_{0}^{2}(t)\right)\right]}
$$

or

$$
c(q)=\exp \left\{-\left[q^{2}(\mathbf{x}(t))-q_{0}^{2}(t)\right] /\left[q_{0}^{2}(t)\left(1+q_{0}^{2}(t)\right)\right]\right\}
$$

and $q(\mathbf{x}(t))$ is named instantaneous coefficient of variation and $q_{0}(t)$ is the speckle scale function. The speckle scale function $q_{0}(t)$ effectively controls the amount of smoothing applied to the image by SRAD.

We observe that in the case of $N$-looks SAR image, we can assume $q_{0}=\frac{1}{\sqrt{N}}$.

The gradient-based speed is computed on the filtered image

$$
v(\mathbf{x}(t))=-\frac{1}{1+\left|\nabla I^{\prime}(\mathbf{x}(t))\right|^{2}}-\lambda k
$$

where $I^{\prime}(\mathbf{x}(t))$ is the image $I(\mathbf{x}(t))$ filtered by SRAD, $k$ is the curvature and $\lambda \in(0,1)$ is a constant. So, the speed term is defined in such a way that the curve proceeds rather fast in low gradient zones, while it wades through to high gradient ones. This strategy allows the contour to propagate until it achieves the limits of the object of interest in the image and then goes slowly close to those 
limits. The implementation obtained by the speed based on image gradient is less accurate in terms of pixels than the previous one, because it works on the filtered image and not on the original one. However, this last approach is independent from the position of the initial curve.

The new velocity is constituted by the mean of the two terms corresponding to the average-based speed and gradient based speed and it is given by a following expression:

$$
v(\mathbf{x}(t))=-\frac{1}{2}\left(\log \frac{\mu_{R_{2}}}{\mu_{R_{1}}}+I(\mathbf{x}(t)) \frac{\mu_{R_{1}}-\mu_{R_{2}}}{\mu_{R_{1}} \mu_{R_{2}}}\right)-\frac{1}{2}\left(\frac{1}{1+\left|\nabla I^{\prime}(\mathbf{x}(t))\right|^{2}}\right)-\lambda k .
$$

As it is possible to see in the (5) the second term is computed on the image filtered by $\operatorname{SRAD~} I^{\prime}(\mathbf{x}(t))$, while the first term is computed on the original image $I(\mathbf{x}(t))$.

The procedure developed by using the combined speed improves the results obtained by the two speeds separately and it saves the property to be independent of the position of the initial curve.

\section{Experimental Results}

To validate the efficiency of the proposed approach, the results obtained applying the combined procedure are compared with ones obtained employing the segmentation process based on the two single speed functions. We tested the procedure on synthetic SAR images to have an exact reference of the contours to detect. Since the location of the edges is not known in the real images and moreover there are not benchmark ones, tests have been synthesized from an original image without noise copying SAR patterns. These tests $(150 \times 150$ pixels) are shown in Figures 1 (a), (b) and (c).

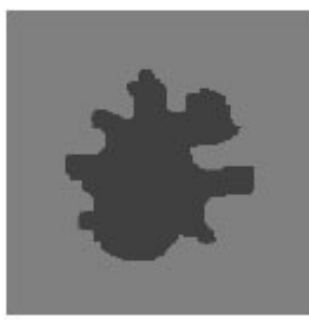

(a) Original image

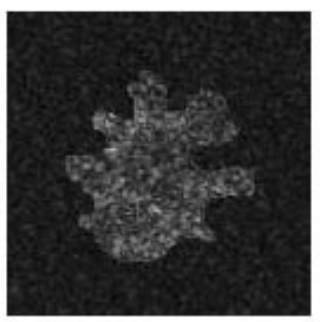

(b) Test image

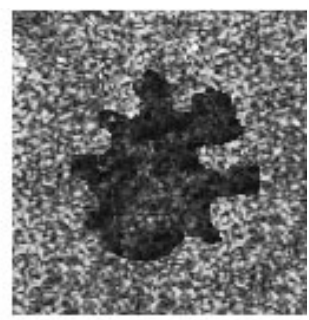

(c) Test image

Fig. 1. Test images

Figures 2 (a), (b), (c), (d) show the initial curves and the corresponding results obtained applying the procedure, using the average-based speed, to the test of Figure 1 (b). We obtain different results starting from different initial contours. In Figures 2 (e), (g) we show the initial contours and the corresponding 
results in (f) and (h), based on the gradient-based speed. In this case the segmentation results do not depend on the initial contours. The final results have been obtained applying the procedure to the smoothed images, by SRAD filter. However, results obtained from both these processes are wrong.

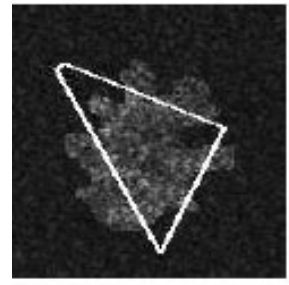

(a) Initial curve

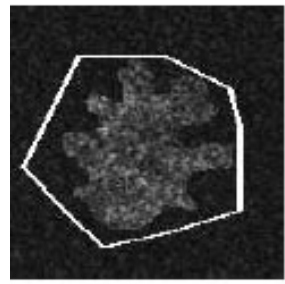

(e) Initial curve

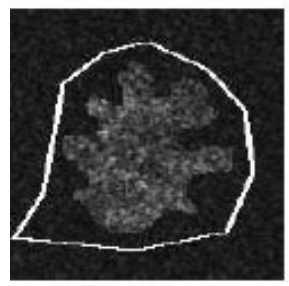

(i) Initial curve

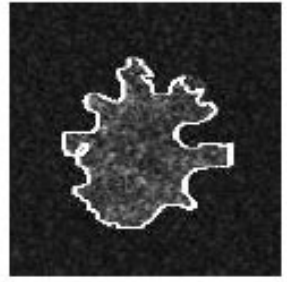

(b) Final curve

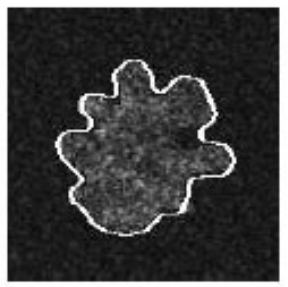

(f) Final curve

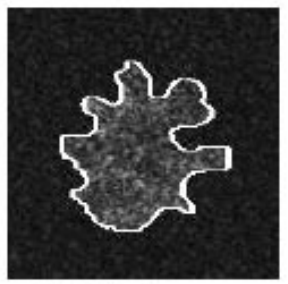

(j) Final curve

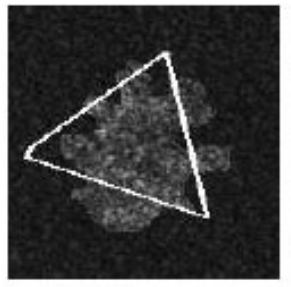

(c) Initial curve

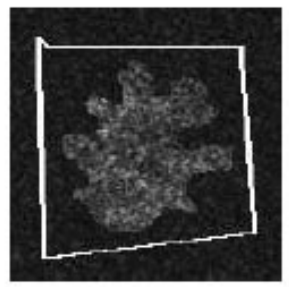

(g) Initial curve

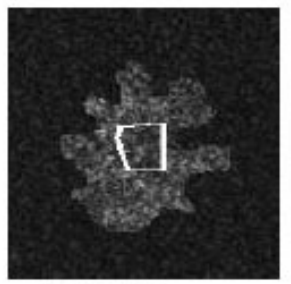

(k) Initial curve

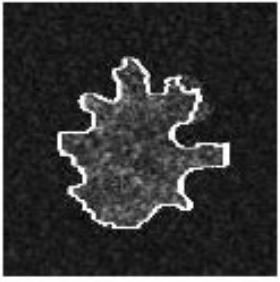

(d) Final curve

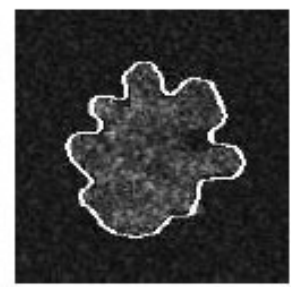

(h) Final curve

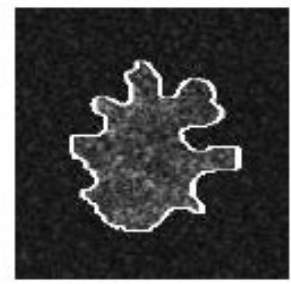

(1) Final curve

Fig. 2. Subplot(a-d) Average-based speed segmentation. Subplot(e-h) Gradient-based speed segmentation. Subplot(i-l) Combined speed segmentation.

The last four Figures show the results, based on the combined speed. In these images the final contours of $(j)$, (l) have been computed starting by both internal and external initial contours of $(\mathrm{j}),(\mathrm{k})$. An important peculiarity of this procedure is that different initial contours lead to the same results.

Analogously, Figure 3 underlines the results obtained applying the segmentation to the image in Figure 1 (c). In particular, Figures 3 (a), (b), (c), (d) show the initial curves and the corresponding results, using the average-based speed. In this case different initial contours lead to different results. Initial contours and the corresponding results, obtained by the gradient-based speed, are shown in Figures $3(\mathrm{e}),(\mathrm{g})$ and $(\mathrm{f}),(\mathrm{h})$ respectively. Here the segmentation result is the 


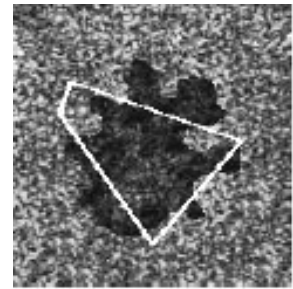

(a) Initial curve

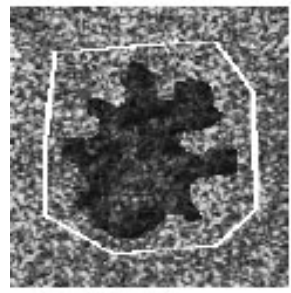

(e) Initial curve

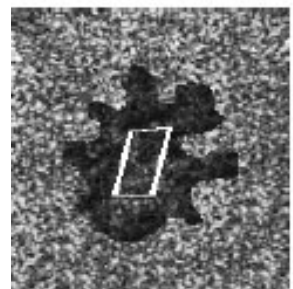

(i) Initial curve

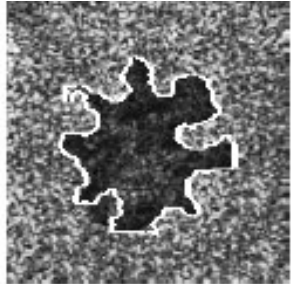

(b) Final curve

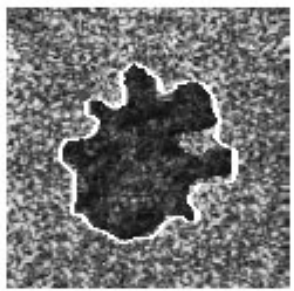

(f) Final curve

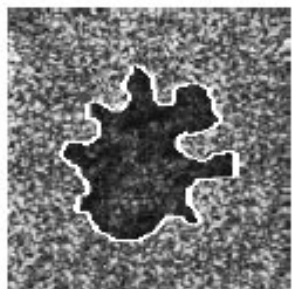

(j) Final curve

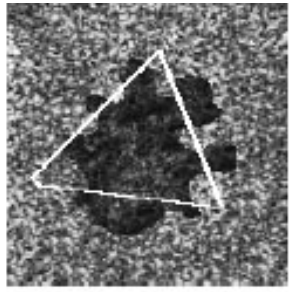

(c) Initial curve

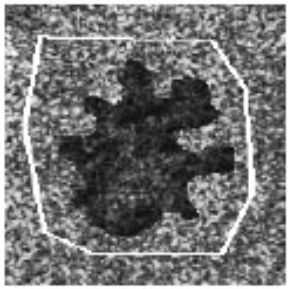

(g) Initial curve

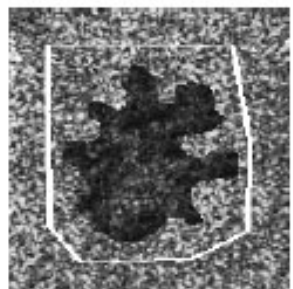

(k) Initial curve

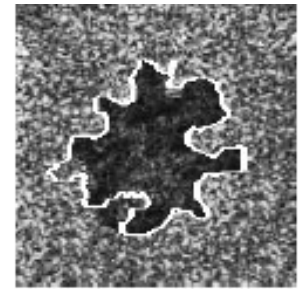

(d) Final curve

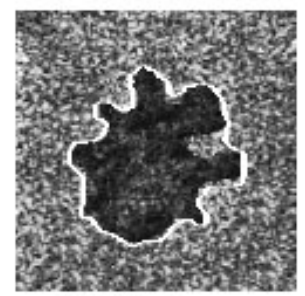

(h) Final curve

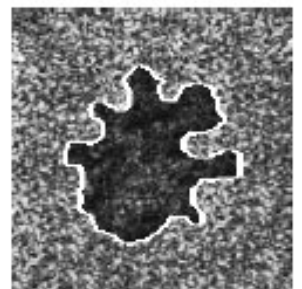

(1) Final curve

Fig. 3. Subplot(a-d) Average-based speed segmentation. Subplot(e-h) Gradient-based speed segmentation. Subplot(i-l) Combined speed segmentation.

same even starting from different initial contours. The final results have been obtained applying the procedure to the filtered images. However, results obtained from both these processes are wrong. The last line of Figure 3 shows the results, based on the combined speed. In these images the final contours of $(\mathrm{j}),(\mathrm{l})$ have been computed starting from both internal and external initial contours of $(\mathrm{j}),(\mathrm{k})$. In this case the same results are obtained starting from different initial contours.

In Figure 4 we present the final contours in (b) and (d) obtained applying segmentation, based on the combined speed, to synthetic images, constituted by disjoint regions, characterized by the same gray levels in (a) and different gray levels in (c).

The segmentation process has also been applied to real images, acquired during ERS2 mission, to extract the contours of the coastlines. In particular, Figure 5 (a) shows the coastline obtained by a $500 \times 700$ pixels SAR image representing the Capraia Island. The convergence is achieved after 3340 iterations. 


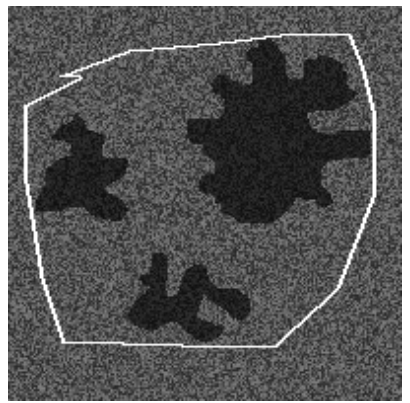

(a) Initial curve

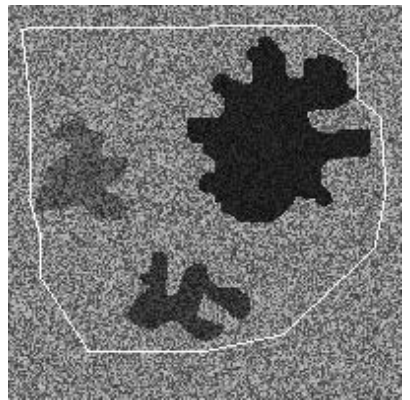

(c) Initial curve

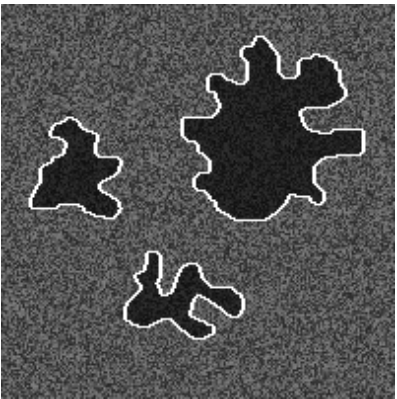

(b) Final curve

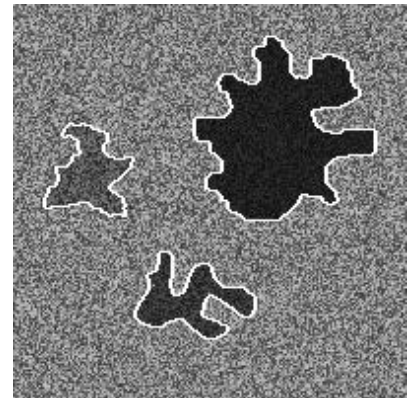

(d) Final curve

Fig. 4. Synthetic image constituted by regions of same gray level (a), regions of different gray level (c), results (b) (d)

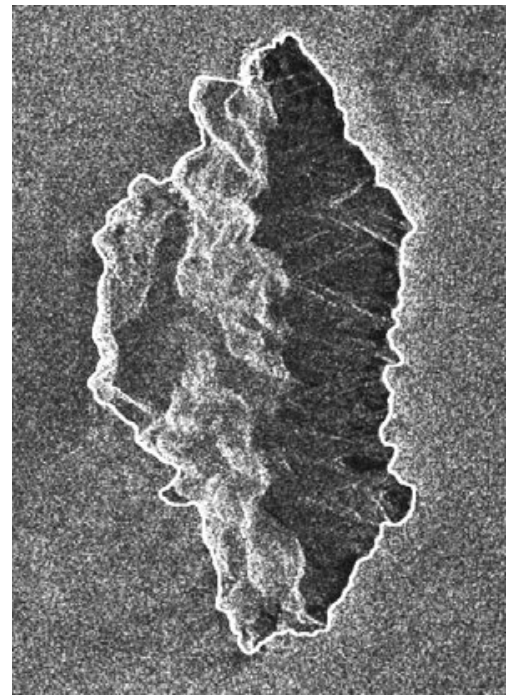

(a) Coast detection from image of Capraia island

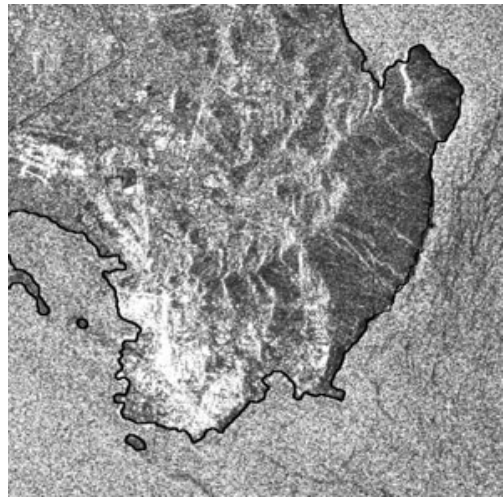

(b) Coast of the Tuscan region

Fig. 5. Original images 


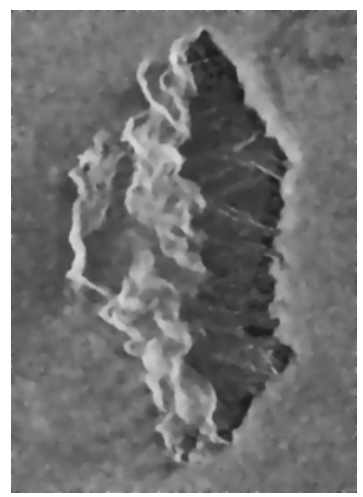

(a) Image of Capraia island smoothed by SRAD

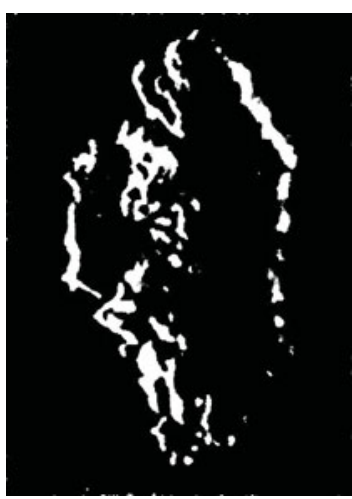

(b) Thresholding technique

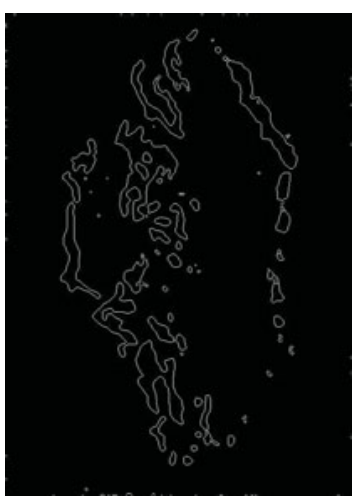

(c) Edge detection technique

Fig. 6. Visual and qualitative comparison with result of Figure 5 (a)

Figure 5 (b) shows the the coastline obtained by a $750 \times 750$ pixels SAR image representing part of the coast of Tuscany. The result shown is obtained after 6500 iterations.

Now we present a visual and qualitative comparison of the results obtained by using segmentation traditional methods. In Figure 6] we show the images produced by applying the technique of segmentation based on the Otzu algorithm and one of edge detection based on the algorithm of Canny. The used images have been smoothed by the SRAD technique.

\section{Conclusions}

In this paper, an approach for SAR image segmentation based on the level set method has been proposed. The procedure has been applied on both synthetic and real SAR images. Segmentation process proposed by us allows the obtained result is independent of the initial location of the curve and moreover, it automatically stops when the curve achieves the boundary. Two distinct speed evolution functions have been examined. The first, based on the mean intensities of the regions, does not need to reduce speckle noise; the second, based on the image gradient, takes into account the problem to filter speckle noise by the SRAD technique. Finally our proposal combining the previous functions improves the results obtained by two individual approaches.

In the future we plan to apply the proposed methodology to images obtained from the constellation of satellites Cosmo Sky-Med. These satellites are useful in monitoring changes in the Earth surface with a very high time resolution, because they can observe the same area several times a day in all weather conditions. Moreover we are interested to extend the procedure to detect the components of the cryosphere such as frozen soil, snow, sea ice, ice sheets from SAR images. 
Acknowledgments. The authors would like to thank Maria Mercede Cerimele for her contribution to this article. Then the authors wish to thank the Consortium for Informatics and Telematics "Innova" of Matera, which has provided the PRI images of ERS Mission.

\section{References}

1. Sethian, J.A.: Level Set Methods and Fast Marching Methods. Cambridge University Press, Cambridge (1999)

2. Sethian, J.A.: Evolution, implementation and application of level set and fast marching methods for advancing front. Journal of Computational Physics 169, 503-555 (2001)

3. Osher, S., Fedkiw, R.: Level Set Methods and Dynamic Implicit Surfaces. Springer, New York (2002)

4. Dellepiane, S., De Laurentiis, R., Giordano, F.: Coastline extraction from SAR images and a method for the evaluation of the coastline precision. Pattern Recognition Letters 25, 1461-1472 (2004)

5. Ben Ayed, I., Mitiche, A., Belhadj, Z.: Multiregion level-set partitioning of synthetic aperture radar images. IEEE Trans. Pattern Analysis and Machine Intelligence 27, 793-800 (2005)

6. Yu, Y., Acton, S.T.: Speckle reducing anisotropic diffusion. IEEE Trans. on Image Processing 11, 1260-1270 (2002)

7. Perona, P., Malik, J.: Scale space and edge detection using anisotropic diffusion. IEEE Trans. Pattern Analysis and Machine Intelligence 12, 629-639 (1990)

8. Cerimele, M., Cinque, L., Cossu, R., Galiffa, R.: Coastline detection from SAR images by level set model. In: Foggia, P., Sansone, C., Vento, M. (eds.) ICIAP 2009. LNCS, vol. 5716, pp. 364-373. Springer, Heidelberg (2009) 